some promise for increased $\mathrm{CO}_{2}$ uptake in managed lands that often receive nutrient or water amendments.

Caution should be used when extrapolating the results from the Minnesotan grasslands to other regions. The grassland biome covers almost $40 \%$ of the global land surface and spans a widely diverse range of soil, plant, and climatic characteristics. An analysis of grassland response to elevated $\mathrm{CO}_{2}$ levels in California, USA, for example, showed little evidence for multiple resource limitation and no effect of elevated $\mathrm{CO}_{2}$ concentrations over five years ${ }^{6}$. In that study, only the addition of nitrogen consistently increased biomass production. The conflicting results across the two studies may have been due to differing plant communities (the Californian grassland was dominated by annual plants versus perennial grassland in Minnesota). It is also possible that other resources (possibly phosphorus) limited the ability of the Californian grassland to take advantage of the higher availability of $\mathrm{CO}_{2}$. These different responses to elevated $\mathrm{CO}_{2}$ highlight the need for more research and continued focus on the mechanisms driving $\mathrm{CO}_{2}$ fertilization.

Reich and colleagues ${ }^{3}$ show that the availability of water and nitrogen in grasslands in Minnesota limit the amount of $\mathrm{CO}_{2}$ uptake by plants over a five-year period. The results imply that $\mathrm{CO}_{2}$ fertilization alone is unlikely to get us off the hook regarding climate change - we can't depend upon natural ecosystems to solve the problem of rising atmospheric $\mathrm{CO}_{2}$ concentrations for us. Instead we need to take a more proactive approach. In addition to investigating the drivers of climate change, more effort should be directed at identifying and testing other land-based solutions, particularly in our managed landscapes.

Whendee L. Silver is in the Department of Environmental Science, Policy, and

Management, University of California, Berkeley, California 94720, USA.

e-mail:wsilver@berkeley.edu

References

1. Norby, R. et al. Proc. Natl Acad. Sci. USA 102, 18052-18056 (2005).

2. Delucia, E. et al. Science 284, 1177-1179 (1999).

Reich, P. B., Hobbie, S. E. \& Lee, T. D. Nature Geosci. 7, 920-924 (2014).

4. Sala, O. E., Parton, W. J., Joyce, L. A. \& Lauenroth, W. K. Ecology 69, 40-45 (1998)

Hooper D. U. \& Johnson, L. Biogeochemistry 46, 247-293 (1999)

6. Dukes, J. S. et al. PLoS Biol. 3, 1829-1837 (2005).

Reich, P. B. et al. Nature 440, 922-925 (2006).

8. Morgan, J. A. et al. Nature 476, 202-205 (2011)

9. http://www.biocon.umn.edu

10. Fowler, D. et al. Phil. Trans. R. Soc. B 368, 20130164 (2013).

Published online: 2 November 2014

\title{
SPACE SCIENCE
}

\section{Astronauts overexposed}

It takes about one year for a spacecraft to travel from Earth to Mars and back. Despite the challenges of such a long voyage, several countries have expressed a long-term goal to send humans to the red planet. However, Nathan Schwadron and colleagues suggest that, because the cosmic radiation environment in the Solar System is steadily deteriorating, a return flight to Mars or beyond may be too long for a human body to safely endure (Space Weather http://doi.org/wfp; 2014).

The current solar cycle has so far displayed the lowest sunspot activity since accurate record keeping began in 1755 . This follows on from abnormally low solar activity during the previous solar cycle (1996-2008) that ended with the longest solar minimum in over 80 years. The pattern of weakening solar activity observed during the current and previous solar cycles is similar to patterns seen in the historical record of sunspot activity during two extreme minima that occurred at the turns of the nineteenth and twentieth centuries, and suggests that solar activity may continue to decline over the next few years.

The decline in solar activity is associated with a weakening of the Sun's magnetic field, which is swept out by the solar wind through the Solar System. This magnetic field shields our Solar System from highenergy radiation - called cosmic rays from elsewhere in the galaxy. Variations in the strength of the Sun's magnetic field

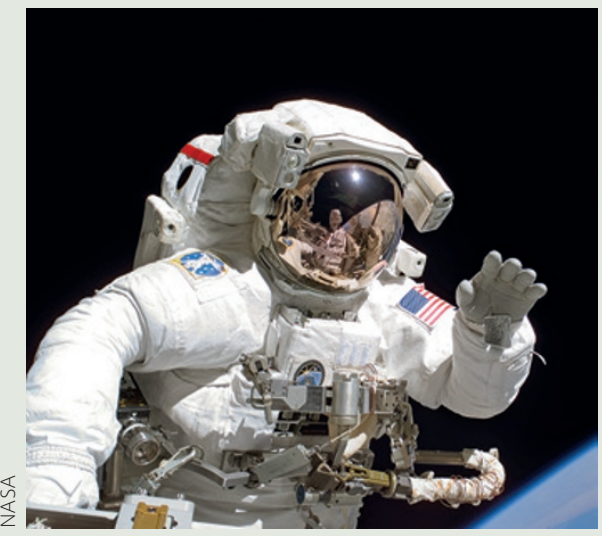

lead to changes in the flux of cosmic rays that reach the inner Solar System. Earth's atmosphere and magnetic field provide a second layer of protection for those on our home planet, but astronauts in interplanetary space are highly susceptible to increases in cosmic ray flux during times of reduced solar activity.

Schwadron and colleagues combine measurements of cosmic ray fluxes collected by the Cosmic Ray Telescope for the Effects of Radiation (CRaTER) instrument onboard NASA's Lunar Reconnaissance Orbiter, with data collected by NASA's Advanced Composition Explorer (ACE) spacecraft to estimate the levels of radiation exposure on the lunar surface and in interplanetary space over the past two solar cycles. Taking into account the thicknesses of aluminium shielding used on typical spacecraft and the interaction of radiation with human tissue, they estimate the dose rate of radiation for a human astronaut.

According to their calculations, the dose rate has increased by about $20 \%$ with each successive solar minimum since the mid1990s. In practical terms, this means that the maximum number of days an astronaut can safely spend in interplanetary space has decreased by this same amount.

Extrapolating their findings into the future, Schwadron and colleagues predict that the allowable time in the increasingly hazardous space radiation environment for a 30-year-old male or female astronaut will be one year or less by the 2020 solar minimum. Thus, a future manned mission to Mars may require thicker shielding on spacecraft and scheduling near solar maximum in order to reduce the radiation risk to within allowable limits - currently defined by NASA as increasing the risk of dying from radiation-induced cancer by no more than $3 \%$.

As the crash of Virgin Galactic's SpaceShipTwo into the Californian Mojave Desert weeks ago demonstrates, just blasting humans into space is fraught with danger. But, once they are there, they face the clock of radiation exposure ticking down - and the amount of time on that clock is getting shorter.

TAMARA GOLDIN 